Document downloaded from:

http://hdl.handle.net/10251/58567

This paper must be cited as:

Ferri García, M.; Camarena Femenia, F.; Redondo, J.; Picó Vila, R.; Avis, MR. (2012).

Explicit finite-difference time-domain scheme for the simulation of 1-3 piezoelectric effect in axisymmetrical configurations. Wave Motion. 49(6):569-584.

doi:10.1016/j.wavemoti.2012.03.007.

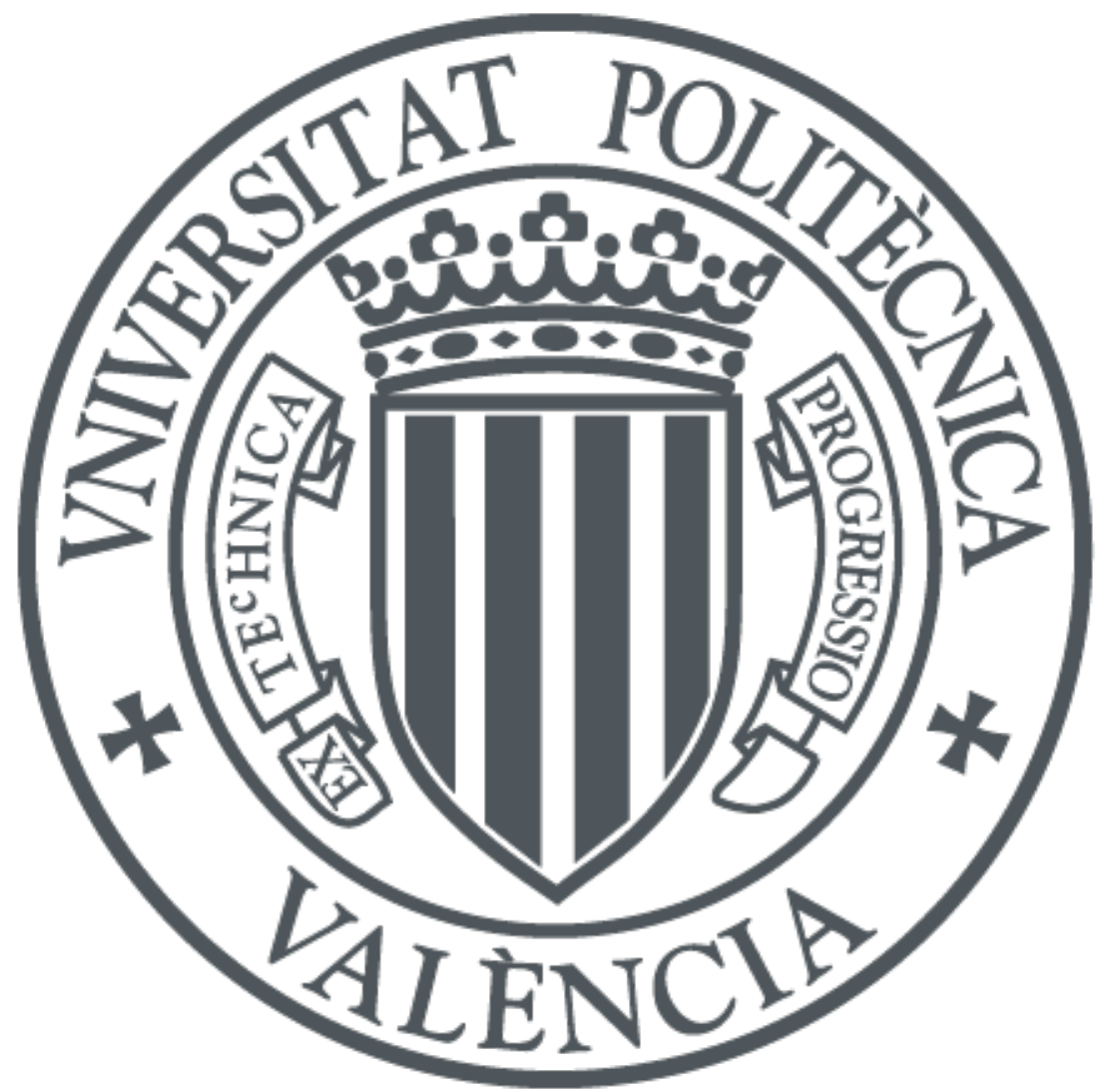

The final publication is available at

http://dx.doi.org/10.1016/j.wavemoti.2012.03.007

Copyright Elsevier

Additional Information 


\title{
Explicit finite-difference time-domain scheme for the simulation of 1-3 piezoelectric effect in axisymmetrical configurations.
}

\author{
Ferri, Marcelino ${ }^{a}$; Camarena, Francisco ${ }^{a}$; Redondo, Javier ${ }^{a}$; Picó, Rubén ${ }^{a}$; Avis, Mark \\ $R^{b}$ \\ a) Institut d'Investigació per a la Gestió Integrada de Zones Costaneres. IGIC. \\ Universitat Politècnica de València, C/Paranimf 1, 46730. Grau de Gandía. Spain . \\ b) Acoustics Research Centre, University of Salford. Newton Building, Greater \\ Manchester, M54WT England.
}

\begin{abstract}
Numerical simulations are useful in the processes of design, development and optimization of transducers for non-destructive testing. In this work, a threedimensional velocity-stress finite-difference model is presented for the elastic wave propagation in the piezoelectric substrate of a transducer excited by applying an impulsive voltage signal to the transducer electrodes. The allocation of the stress, velocity and electric fields components on a staggered grid leads to a stable scheme. The different time scales of both mechanical and electromagnetic waves have leaded previous FDTD models to choose between significant physical simplifications or complicated implicit equations. The model presented here is explicit in all its time domain equations, contains only first order derivatives and is centered in time and space. The results of simulations show remarkable accuracy and stability for the different transducers studied.
\end{abstract}

\section{Keywords}

Wave propagation, coupled electromechanic field, time domain piezoelectric simulation

\section{1.- INTRODUCTION}

An algorithm to simulate elastic wave phenomena in cylindrical piezoelectric transducers is presented in this paper. In the piezoelectric substrate, electromagnetic and acoustic fields are coupled, so wave dynamics present multiple time scales with notably different wave speeds. In order to resolve these multiple time scales (essential for numerical stability), the fastest time scale must be respected. Generic multiphysics packages may have this capability, but in order to be foolproof, they implement stiff system solvers as the default. Hence a multiphysics package will give an incorrect solution for the coupled wave dynamics problem unless the modeler knows and respects the time scale of the fastest physical mode. Furthermore, given the different order of magnitude of both time scales, and taking into account the significant impact of the wave speed with respect to stability and computational cost criteria, it is preferable to assume or propose one of the so-called electromagnetic quasi-static approaches when our interest is focused on mechanical waves.

The only obligatory condition of the general quasi-static approach is that the size of the piezo-substrate must be considerably smaller than the wavelength of electromagnetic phenomena within the simulation. However, these requirement usually introduce additional significant simplifications to enable the development of practical models [1- 
4], and thus it is not difficult to find case studies where predicted behavior differs considerably from that observed in practice. Most of the previous proposed models [e.g. 1-11], whilst achieving satisfactory results, also assume that one of the electrical vectors (electrostatic field or displacement) must be uniform and sometimes constant within the domain. The selection of the vector to be fixed depends on the electrical contour condition, and therefore open circuit and short-circuit conditions are respectively confused with fixed displacement and fixed electrical field, neglecting the piezoelectric coupling. Thus, for many specific cases, less restrictive approaches must be developed.

In recent years the works of Smith and Ren [2] or Chagla and Smith [3] may be noted; they impose the condition that all electrical rotationals must be null, implying that the electrical displacement into the piezoelectric must be uniform. Other more accurate conditions are proposed by Wong and Tam [4], but their work cannot be considered as a complete explicit FDTD implementation; in fact, they state that an external "simulator named ANSOFT Maxwell 2D is used to calculate the electric displacement D within the substrates". A better approach is achieved by Gsell and Dual [5] and May and Dual [6], but both models are explicit for all the variables with the exception of the electrostatic potential. To the best of our knowledge, the only other recent significant contributions on this topic come from Masahiro Sato et al. [7,8], whose work has achieved important advances but is restricted to the two dimensional case. Other important work to be noted, in which no restrictive conditions are imposed is that of Hornsby and Das-Gupta [9].

The aim of this work is to define a quasi-static approach compatible with an accurate physical definition of the problem and with a computational full explicit implementation. Thus we assume as usual that all piezo-substrate dimensions are smaller than the wavelength of any electromagnetic field, but we allow both displacement and electric fields to be variable in time and space. As will be shown, the critical point is to calculate explicitly the electrical displacement in each computational node. To facilitate this task, the simplification proposed here consists on accepting that electrical displacement is uniform along an axial field line, but dependent on time and radial direction within the piezosubstrate.

The required explicitness needs different sets of equations, depending on the application of the piezoelectric material; i.e. different set for actuators rather than for sensors, but also different for a short-circuited sensor than for an open-circuited or large-impedance device. A complete multipurpose algorithm will be developed in due course, but in this paper, for the sake of simplicity and clarity, we will start by presenting the simplest case: actuators with external voltage excitation function.

The equation system is presented as a two dimensional computational domain which represents a physical 3D axisymmetrical device. The selection of this symmetry is due to computational limitations which persist for full 3D simulations even when using recent high-spec machines. Since many practical piezoelectric ceramic devices display axial symmetry, we consider that axisymmetrical equations are directly applicable to real problems, whereas 2D Cartesian simulations are less amenable to comparison with real devices.

The authors are conscious that the stated problem has been approached successfully using FEM (finite element method) schemes [12-15]; however, the ease of model 
construction and availability of direct time domain calculations when using FDTD models justifies their introduction for many particular case studies. In fact, in echoimpulse techniques and directional radiation problems, the time domain knowledge of physical variables is a priority, and it is difficult to obtain them from the inverse-Fourier transform of numerically-obtained eigenvalues.

\section{2.- NOTATION}

$\rho_{o}:$ Volumetric mass density in equilibrium

$\rho_{V}$ : Volumetric charge density

$\rho_{S}$ : Surface charge density

$\vec{E}$ : Electric field, with compounds $E_{i}$

$\vec{D}$ : Electric displacement, with compounds $D_{k}$

$\vec{H}$ : Magnetization

$\vec{B}:$ Magnetic field

$\vec{j}$ : Current density

$\phi$ : Electrostatic potential

$\vec{u}$ : punctual instantaneous speed, with compounds $u_{i}$

$\vec{\xi}$ : punctual deformation from equilibrium, with compounds $\xi_{i}$

$\sigma_{i j}$ compounds of the stress tensor

$s_{i j}=$ compounds of the unitary strain tensor, in Cartesian coordinates

$s_{i j}=\frac{1}{2}\left(\frac{\partial \xi_{i}}{\partial x_{j}}+\frac{\partial \xi_{j}}{\partial x_{i}}\right)$

$k_{i j}^{D}$ Compounds of the elasticity tensor in conditions of constant electric displacement

$h_{k i}$ : piezoelectric modulus which defines the linear dependence between $\sigma_{i}$ and $D_{k}$, and also between $E_{k}$ and $s_{i}$, being $s_{i}$ and $\sigma_{i}$ expressed in reduced form (as noted in next paragraph about conventions)

$\beta_{k k}^{S}$ Inverse of absolute electric permittivity in condition of constant deformation

\section{1.-Conventions}

Coordinates

Following usual conventions, in equations written in cylindrical coordinates we will use $x_{1}, x_{2}, x_{3}$ to refer to the components $r, \varphi, z$

Vectorial variables: Example electric field E,

$E_{r} \equiv E_{1}, E_{\varphi} \equiv E_{2}, E_{z} \equiv E_{3}$ in cylindrical

$E_{x} \equiv E_{1}, E_{y} \equiv E_{2}, E_{z} \equiv E_{3}$ in Cartesian

The axisymmetrical axis $\mathrm{z}$ will always be denoted by the sub index 3 , as conventional in piezoelectricity 
Tensorial variables

Strain and stress tensors notation will be reduced, as follows,

$\sigma_{r r} \equiv \sigma_{1}, \sigma_{\varphi \varphi} \equiv \sigma_{2}, \sigma_{z z} \equiv \sigma_{3}, \sigma_{r z} \equiv \sigma_{5}$, in cylindrical

$\sigma_{x x} \equiv \sigma_{1}, \sigma_{y y} \equiv \sigma_{2}, \sigma_{z z} \equiv \sigma_{3}, \sigma_{y z} \equiv \sigma_{4}, \sigma_{x z} \equiv \sigma_{5}, \sigma_{x y} \equiv \sigma_{6}$, in Cartesian

The same reduction is carried out for strain $s$.

Note that symmetry restrictions imply that tensorial compounds $\sigma_{r \varphi}, \sigma_{\varphi z}$ vanish.

\section{3.- SCOPE OF THE MODEL}

When a sample of piezoelectric material is mechanically excited and no electrodes are present, mechanical waves propagate like in any elastic material. The absence of electrodes implies a null displacement field in the whole piezoelectric region, whereas the electric field can vary associated with perturbation. In fact, neglecting the displacement field in canonical equations (20-23), this set of equations -and therefore the dynamics of the system- becomes identical to that of any elastic solid [16]. Thus, the presence of electrodes is responsible for generating electrical charges, displacement fields and the modification of piezoelectric dynamics in comparison with simple elastic solids. It will be understood that the larger the influence of electrodes, the more complex the dynamics in comparison with a simple elastic case.

The system is capable to simulate configurations where the electrode is fully integrated with the elastic piezostructure, such as transducers. For the sake of simplicity, we present and evaluate the system for the case of a cylinder with radius R and thickness $Z$, excited by flat electrodes attached to the lateral faces (Figure 1).

The model can easily be implemented to simulate configurations where other media, different than the piezoelectric substrate, are present. In fact, the system and the equations are targeted to be useful to simulate time domain directivity patterns generated by piezoelectric transducers radiating inside a solid or fluid. In this kind of situations, implicit models [4-6] present numerical difficulties (due to the radiation in an open media), and previous explicit approaches [2,3] assume more restrictive hypothesis.

\section{4.- GOVERNING EQUATIONS OF PIEZOELECTRIC SYSTEMS.}

The general set of equations involved, which later will be particularized to the axisymmetrical case (with axial symmetry required in shape, excitation, and piezoelectric isotropy) is presented. On the one hand, the Maxwell equations for the electromagnetism

$$
\nabla \times \vec{E}=-\dot{\vec{B}}, \nabla \times \vec{H}=\vec{j}+\dot{\vec{D}}, \nabla \cdot \vec{D}=\rho_{V}, \nabla \cdot \vec{B}=0
$$

where variables are defined above,

and, on the other hand, "mechanical" equations, consisting on the stress equation of motion and the canonical constitutive equation. For the linear undamped case, without 
convective terms considered, they could be written respectively as follows (in matrix form for Cartesian coordinates):

$$
\begin{gathered}
\frac{\partial}{\partial t}\left(u_{1}, u_{2}, u_{3}\right)=\frac{1}{\rho_{o}}\left(\frac{\partial}{\partial x_{1}}, \frac{\partial}{\partial x_{2}}, \frac{\partial}{\partial x_{3}}\right)\left(\begin{array}{lll}
\sigma_{11} & \sigma_{12} & \sigma_{13} \\
\sigma_{21} & \sigma_{22} & \sigma_{23} \\
\sigma_{31} & \sigma_{32} & \sigma_{33}
\end{array}\right) \\
\left.\left(\begin{array}{l}
\sigma_{1} \\
\sigma_{2} \\
\sigma_{3} \\
\sigma_{4} \\
\sigma_{5} \\
\sigma_{6}
\end{array}\right)=\left(\begin{array}{llllll}
k_{11}^{D} & k_{12}^{D} & k_{13}^{D} & 0 & 0 & 0 \\
k_{21}^{D} & k_{22}^{D} & k_{23}^{D} & 0 & 0 & 0 \\
k_{31}^{D} & k_{32}^{D} & k_{33}^{D} & 0 & 0 & 0 \\
0 & 0 & 0 & k_{44}^{D} & k_{45}^{D} & 0 \\
E_{2} \\
E_{3}
\end{array}\right)\left(\begin{array}{llllll}
k_{54}^{D} & k_{55}^{D} & 0 \\
0 & 0 & 0 & 0 & 0 & k_{66}^{D}
\end{array}\right) \quad-\left(\begin{array}{lllll}
h_{11} & h_{21} & h_{31} \\
h_{12} & h_{22} & h_{32} \\
h_{13} & h_{23} & h_{33} \\
h_{14} & h_{24} & h_{34} \\
h_{15} & h_{25} & h_{35} \\
h_{16} & h_{26} & h_{36}
\end{array}\right)\right) \quad\left(\begin{array}{l}
s_{1} \\
s_{2} \\
s_{3} \\
s_{4} \\
s_{5} \\
s_{6}
\end{array}\right) \\
\left.-\left(\begin{array}{lllllll}
h_{12} & h_{13} & h_{14} & h_{15} & h_{16} \\
h_{21} & h_{22} & h_{23} & h_{24} & h_{25} & h_{26} \\
h_{31} & h_{32} & h_{33} & h_{34} & h_{35} & h_{36}
\end{array}\right)\left(\begin{array}{ccc}
\beta_{11}^{s} & 0 & 0 \\
0 & \beta_{22}^{s} & 0 \\
0 & 0 & \beta_{33}^{s}
\end{array}\right)\right) \quad\left(\begin{array}{l}
D_{1} \\
D_{2} \\
D_{3}
\end{array}\right)
\end{gathered}
$$

The complete solution of the presented set of equations includes electromagnetic wave propagation. To ensure numerical stability of a hypothetical complete model, the time increment in each computational step becomes very small, enforcing low numerical accuracy in the propagation of mechanical waves and wasting significant computational resources. Thus, if we restrict the analysis to piezoelectric substrates with much smaller dimensions than the wavelength of the electromagnetic waves in the bandwidth of interest, we can introduce a quasi-static approach for which $\nabla \times \vec{E}=0$. Then, the electrostatic potential $\phi$ can be defined as:

$$
\vec{E}=-\nabla \phi
$$

For our purposes, equation (2) will not be reduced to $\nabla \times \vec{H}=0$, as usual in a quasistatic approach ([2-3]). Instead, we propose a less restrictive simplification (see appendix B for more detailed justification) relative to the electrical displacement vector field; that is, for the particular case of axial symmetry:

$$
\begin{aligned}
& |\vec{D}|=D_{z}, \text { and } \\
& D_{z}=0 \text { for } r>R
\end{aligned}
$$

This means that the value of $\boldsymbol{D}$ outside the substrate is negligible in comparison with its value within it; i.e. all the electrical flux is concentrated in the ferroelectric material. This approach becomes more accurate the smaller the ratio between the $z$ and $r$ dimensions is (condition that is usually fulfilled in sandwich transducer configurations). The proposed approach, given in equation (8), joined to Gauss law for the displacement field $\left(\nabla \cdot \vec{D}=\rho_{V}\right)$, implies that the electric displacement $D_{z}$ remains constant over any line parallel to the symmetry axis, since there are no free electrical charges within the piezoelectric substrate. Thus, if we evaluate the instantaneous difference of potential between two points of the opposite electrodes $\phi_{t o t}(r)$ in a line parallel to the third axis, we find: 


$$
-\phi_{t o t}(r)=-\phi_{t o t}\left(x_{1}\right)=\int_{o}^{Z} E_{3}\left(x_{1}, x_{3}\right) d x_{3}=\int_{o}^{Z}\left(-h_{3 j} s_{j}\left(x_{1}, x_{3}\right)+\beta_{33}^{s} D_{3}\left(x_{1}\right)\right) d x_{3}
$$

where the summation convention holds for the index $j$ and $Z$ is the axial distance between electrodes

The previous equation, which can be written

$$
-\phi_{t o t}\left(x_{1}\right)=\int_{o}^{Z}\left(-h_{3 j} s_{j}\left(x_{1}, x_{3}\right)\right) d x_{3}+Z \beta_{33}^{s} D_{3}\left(x_{1}\right),
$$

operates in the simulation as an electrical boundary condition. In fact, given a known value of the potential between the electrodes, it defines the value of the displacement for each line $D_{3}\left(x_{1}\right)$ which is coincident-given the approach stated in equations (8) and (9)- with the surface density charge in each point of the electrode $\rho_{S}\left(x_{1}\right)$.

Equation 11, joined with equations 5 and 6 and mechanical boundary conditions in cylindrical coordinates, configure the whole explicit FDTD model. The first timederivative of canonical equation in cylindrical coordinates with axial symmetry reads:

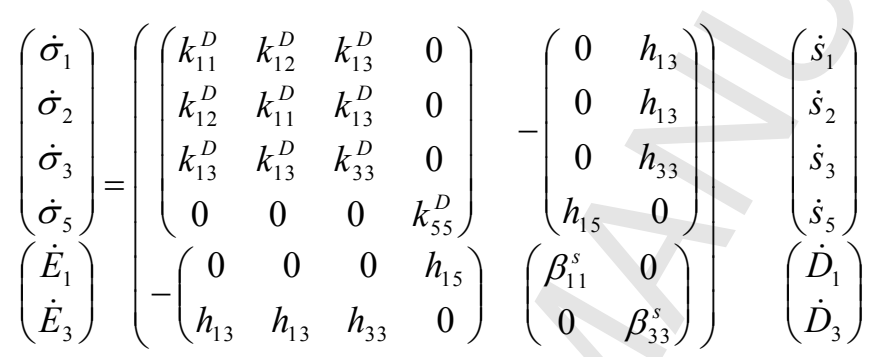

Where the total number of elastic modules have been reduced, and only those independent in axisymmetrical anisotropy have been written. Mechanical, electrical and piezoelectric modules have been considered as time-independent. Finally, the variable compounds $\dot{E}_{2}, \dot{D}_{2}, \dot{s}_{4}, \dot{s}_{6}, \dot{\sigma}_{4}$, and $\dot{\sigma}_{6}$ are null due to axial symmetry, and have therefore been excluded from the equations.

The time derivative of the strain tensor, $\dot{S}$, is related with the tensor of the velocity gradient, $\nabla u$, as follows

$$
\dot{S}=\frac{1}{2}\left(\nabla u+(\nabla u)^{T}\right)
$$

where this tensor in Cartesian coordinates is $(\nabla u)_{i j}=\partial u_{j} / \partial x_{i}$, and in cylindrical coordinates takes the form

$$
\nabla u=\left(\begin{array}{ccc}
\frac{\partial u_{r}}{\partial r} & \frac{\partial u_{\varphi}}{\partial r}-\frac{u_{\varphi}}{r} & \frac{\partial u_{z}}{\partial r} \\
\frac{1}{r} \frac{\partial u_{r}}{\partial \varphi} & \frac{1}{r} \frac{\partial u_{\varphi}}{\partial \varphi}+\frac{u_{r}}{r} & \frac{1}{r} \frac{\partial u_{z}}{\partial \varphi} \\
\frac{\partial u_{r}}{\partial z} & \frac{\partial u_{\varphi}}{\partial z} & \frac{\partial u_{z}}{\partial z}
\end{array}\right)
$$


Thus, we obtain

$$
\dot{s}_{1}=\frac{\partial u_{r}}{\partial r}, \quad \dot{s}_{2}=\frac{1}{r} \frac{\partial u_{\varphi}}{\partial \varphi}+\frac{u_{r}}{r}, \quad \dot{s}_{3}=\frac{\partial u_{z}}{\partial z}, \quad \dot{s}_{5}=\frac{1}{2}\left(\frac{\partial u_{z}}{\partial r}+\frac{\partial u_{r}}{\partial z}\right)
$$

where axial symmetry restrictions reduce the equation for the second compound to $\dot{s}_{2}=u_{r} / r$. Finally, the time-derivative constitutive equations in cylindrical coordinates are as follows

$$
\begin{aligned}
& \dot{\sigma}_{1}=k_{11}^{D} \frac{\partial u_{r}}{\partial r}+k_{12}^{D} \frac{u_{r}}{r}+k_{13}^{D} \frac{\partial u_{z}}{\partial z}-h_{13} \dot{D}_{3} \\
& \dot{\sigma}_{2}=k_{12}^{D} \frac{\partial u_{r}}{\partial r}+k_{11}^{D} \frac{u_{r}}{r}+k_{13}^{D} \frac{\partial u_{z}}{\partial z}-h_{13} \dot{D}_{3} \\
& \dot{\sigma}_{3}=k_{13}^{D} \frac{\partial u_{r}}{\partial r}+k_{13}^{D} \frac{u_{r}}{r}+k_{33}^{D} \frac{\partial u_{z}}{\partial z}-h_{33} \dot{D}_{3} \\
& \dot{\sigma}_{5}=\frac{k_{55}^{D}}{2}\left(\frac{\partial u_{z}}{\partial r}+\frac{\partial u_{r}}{\partial z}\right)-h_{15} \dot{D}_{1} \\
& \dot{E}_{1}=-\frac{h_{15}}{2}\left(\frac{\partial u_{z}}{\partial r}+\frac{\partial u_{r}}{\partial z}\right)+\beta_{11}^{s} \dot{D}_{1} \\
& \dot{E}_{3}=-h_{13} \frac{\partial u_{r}}{\partial r}-h_{13} \frac{u_{r}}{r}-h_{33} \frac{\partial u_{z}}{\partial z}+\beta_{33}^{s} \dot{D}_{3}
\end{aligned}
$$

The stress equation of motion takes the form (see ref [16]):

$$
\begin{aligned}
\frac{\partial u_{r}}{\partial t} & =\frac{1}{\rho_{o}}\left(\frac{1}{r} \frac{\partial\left(r \sigma_{r r}\right)}{\partial r}+\frac{\partial \sigma_{r z}}{\partial z}-\frac{\sigma_{\varphi \varphi}}{r}\right) \\
\frac{\partial u_{z}}{\partial t} & =\frac{1}{\rho_{o}}\left(\frac{1}{r} \frac{\partial\left(r \sigma_{r z}\right)}{\partial r}+\frac{\partial \sigma_{z z}}{\partial z}\right)
\end{aligned}
$$

where the equation for the second component has been excluded, since with axial symmetry $\frac{\partial u_{\varphi}}{\partial t}=0$.

Previous eight equations and the time derivative of equation (11) are implemented in the numerical model as shown in next section. This time derivative in cylindrical coordinates reads:

$$
\dot{D}_{3}\left(x_{1}\right)=\frac{1}{Z \beta_{33}^{s}}\left(\int_{o}^{Z}\left(h_{13} \frac{\partial u_{r}}{\partial r}+h_{13} \frac{u_{r}}{r}+h_{33} \frac{\partial u_{z}}{\partial z}\right) d x_{3}-\dot{\phi}_{t o t}\left(x_{1}\right)\right)
$$




\section{5- DISCRETIZATION OF THE EQUATIONS.}

The canonical equations (20-25) have been chosen in a manner which makes it easy to reduce the system to pure mechanics. In fact, as stated in the introduction, in the case that $\boldsymbol{D}$ is forced to be constant in the complete computational domain, the actual set of equations $(20-23,26-27)$ becomes entirely equivalent to that proposed by Schubert for solids [16]. Furthermore, the term associated with $\dot{D}_{3}$ in equations (20-22) is formally a source term, so if we impose a known function for $\dot{D}_{3}$, the piezoelectric substrate vibrates in exactly the same way as a general solid with the same source function.

The coupled piezoelectric effect in this model of an actuator is given by the "electrical boundary condition" in Eq (28). Starting from an imposed known potential function between the electrodes, this equation determines the electric displacement for each instant and position. This electric displacement is obtained instantaneously as a function of the external electric conditions and the mechanical punctual state. Thus, it is the variable that takes into account the electro-mechanical full coupling, receiving the entire electromechanical prior history and acting like a source term in the next time step.

To describe the explicit FDTD scheme let us consider a piezoelectric cylinder with radius $R$ and thickness $Z$ excited by two flat electrodes attached to the opposite sides of the cylinder. Stress-free mechanical boundary conditions are imposed. The computational domain defined for the physical configuration is a rectangular mesh with $J$ and $K$ nodes respectively in radial and axial directions (see Figure 1). Note that on the periphery, for some nodes ("phantom nodes") artificial conditions are forced in order to obtain significant values of the physical magnitudes in the real contour. Both layers (real boundary and the phantom nodes) are required due to the second order nature of the general motion equations.

From the mechanical equations $(20-23,26-27)$ we note that time derivatives of the speed, $\vec{u}$, are computed as spatial derivatives of the stress, $\sigma$, and vice versa. This particularity of mechanical, and also electromagnetic equations, suggests a leapfrog time-step structure, as is commonly used in explicit FDTD schemes. With this strategy, a set of variables $(\sigma, E, D)$ is computed at odd half-time intervals and the rest $(\vec{u})$ at even half-time intervals.

An appropriate spatial staggered scheme is defined. In the scheme proposed (represented in Figure 1) different components of each vectorial or tensorial variable may be placed at different nodes of the spatial mesh. The computational evolution of calculation consists of three basic steps, two of which are coincident with time steps and the third is performed without "physical time advance"(see Figure 2).

The scheme is therefore centered in space and time. This fully centered first order scheme represents a computational advance regarding to higher order systems with second order derivatives and a non-centered time scheme, where approximated methods (like Runge-Kutta) would be necessary. The accuracy of the algorithm is dependent on the validity of the discretisation approach, which approximates mathematical differentials by finite differences. The whole set of discrete equations, for substrate and boundaries, is given in the appendix A. 


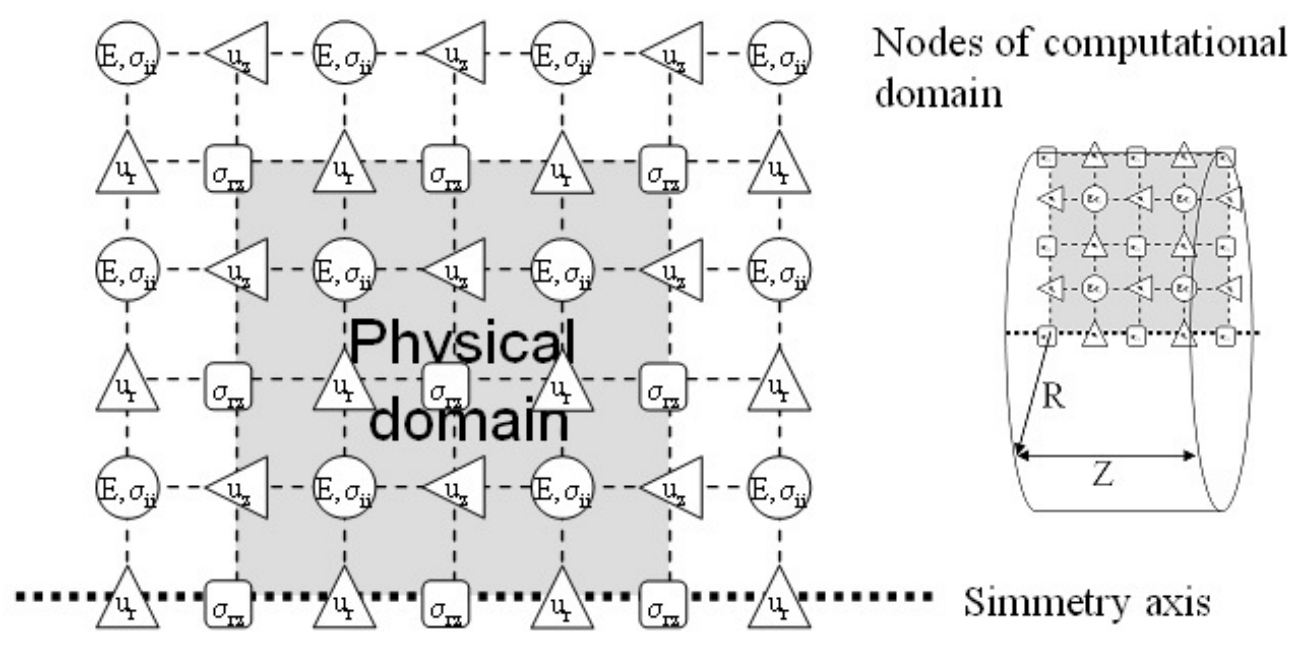

Figure 1.- Structure of the staggered spatial mesh

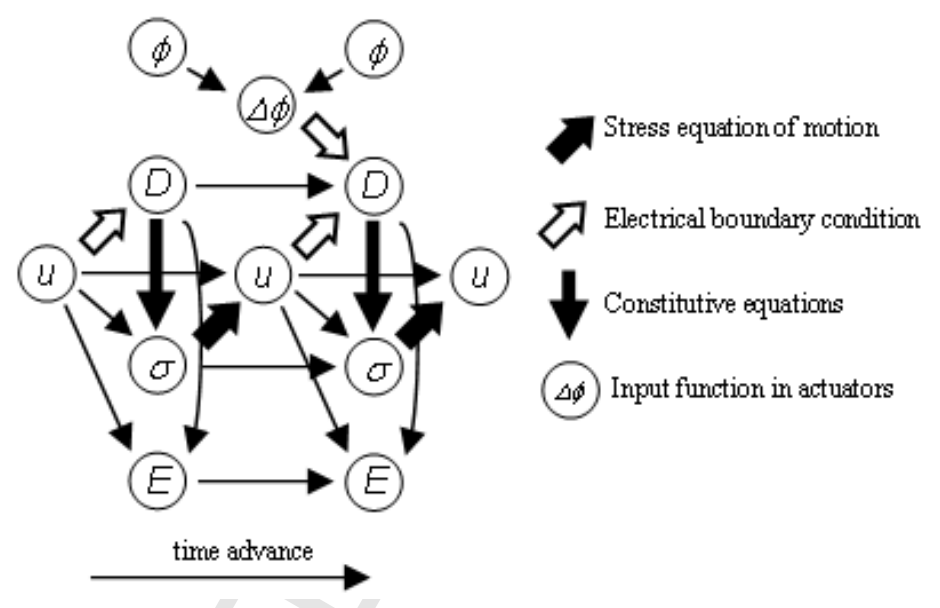

Figure 2.- Time and computational evolution of the simulation.

\section{1.- Stability}

The selection of the time step attending to the Courant number [17] has proved sufficiently to avoid numerical instability. No difficulties were encountered, since the constitutive equations are defined in terms of $k^{D}$ coefficients which yield to wave speeds bigger than those defined from $k^{E}$ coefficients. Thus, time discretisation is restricted by the size of the shell and by the highest longitudinal wave speed, $c_{\text {max }}=\sqrt{k_{\max }^{D} / \rho_{o}}$, given the next relation:

$$
\Delta t \leq \frac{1}{c_{\max } \sqrt{1 / \Delta r^{2}+1 / \Delta z^{2}}}
$$


where $\Delta t, \Delta r$ and $\Delta z$ are respectively the time step and the spatial step in the respective directions. If a quadratic grid is used, $\mathrm{Eq}(32)$ reduces to

$$
\Delta t \leq \frac{\Delta z}{c_{\max } \sqrt{2}}
$$

It is well known that the smaller the relative size of the space step with respect to wavelength, the better the accuracy. The size of the mesh in all the simulations of this work has been chosen sufficiently small. Particularly

$$
\Delta z<<\frac{c_{\min }}{8 f_{\max }}
$$

where $f_{\max }$ denotes the highest frequency evaluated in the signal, and $c_{\min }$ is the lowest wave speed of the piezoelectric domain.

\section{6- NUMERICAL RESULTS AND DISCUSSION}

In this section we will present some numerical experiments. The geometry and numerical parameters are the following: A piezoelectric cylinder of variable radius $(R=1 \mathrm{~mm}$ to $R=40 \mathrm{~mm})$ and length $(\mathrm{Z}=1 \mathrm{~mm}$ to $\mathrm{Z}=60 \mathrm{~mm})$. The material consist of axially polarized PZ21 from Ferroperm, with volumetric mass density $7500 \mathrm{kgm}^{-2}$, and piezoelectrical properties listed in table 1 . The substrate principal direction is placed parallel to the cylinder axis to fulfill the condition of axial symmetry in both piezoelectric isotropy and geometry. Two flat electrodes are attached to both flat cylinder ends, and the excitation external voltage functions are Ricker wavelets (to obtain frequency plots via the Fourier transform) and harmonic pure tones modulated by a Hanning function (to obtain instantaneous snapshots). The central frequency and bandwidth of the Ricker wavelets may vary depending on the region where the eigenvalues of the system are expected. The mesh is composed by nodes separated $0.1 \mathrm{~mm}$ in radial and axial direction, and different time steps have being tested, satisfying stability condition, in the interval between $15 \mathrm{~ns}$ to $25 \mathrm{~ns}$.

\section{1-Frequency response}

Analytical solutions are known for the more straightforward geometries in elastic solids. Eigenfrequencies associated with pure axial and radial modes are defined by known analytical expressions. These eigenfrequencies are dependent on the wave speed of the perturbation and geometrical dimensions of the solid. However, one of the main differences between piezoelectrics and elastic solids is that the first ones are capable to develop different propagation speeds -as a function of applied electromagnetic conditions- being those speeds defined between the two limit wave speeds, $c^{E}$, the slowest one, associated with the constant electric field condition, and $c^{D}$, the fastest one, associated with the constant electric displacement condition.

As a consequence of this particularity, the vibratory behavior of piezos and elastic solids are generally non equivalent; but there are certain particular combinations of geometry and electromagnetic conditions in piezoelectric transducers that lead to a vibration in the piezo quite similar to the vibration of simple elastic solids.

More specifically, it can be demonstrated that a very thin disc excited in short circuit vibrates in its radial modes in a similar way to an elastic solid with identical shape and wave speed $c^{E}$. By the other hand, a long and thin rod excited in open circuit condition 
vibrates -in its axial modes- similarly to a simple elastic solid with identical shape and wave speed $c^{D}$.

We can justify previous statement as follows: In the case of the thin disc vibrating in a radial resonant mode, it can be accepted that the perturbation along an axial line remains practically constant. Thus, the null electrostatic potential condition (shortcircuit) is, as a first approach, similar to the null electric field condition, whose associated wave speed is $c^{E}$. In the case of the long and thin rod vibrating in an axial resonant mode, it can be accepted that perturbation along a radial line remains practically uniform. Thus, the null electric current condition (open circuit) is, as a first approach, similar to the null electric displacement condition, whose associated wave speed is $c^{D}$.

In the next paragraphs we show the results of the evaluation in the aforementioned limit cases, i.e., short circuited thin disk and open circuit thin rod. Comparison has been performed between numerical model response and analytical solution of the correspondent elastic solid. Eigenfrequencies obtained show that the model is able to perform in both limit cases with the respective different limit wave speeds, characteristic of wave propagation in piezos, $c^{E}$ and $c^{D}$. This adequate response in limit situations, joined with the coherence and stability of model equations, allow as adventure a correct behavior in intermediate situations.

In order to obtain eigenfrequencies numerically we compute instantaneous values of electric voltage and current in the electrode, calculated from an axial spatial integration of the whole domain, and from a radial surface integration of the whole domain respectively. Once obtained these time domain functions, we calculate the complex fourier transform of them, and finally, the frequency domain impedance and admittance from the division of both frequency domain functions. The plots show the absolute values of admittance and impedance. The resonant and anti-resonant frequencies are obtained from the local maxima of these functions. Notice that both impedance and admittance functions are defined in the frequency domain for electrical signals, and we have obtained them from a time domain simulation of system mechanics, therefore, all numerical accumulated errors should appear on the plots. If no significant lack of precision is found here, we can be confident concerning the accuracy of the entire time domain simulation.

Very good agreement is found when comparing analytical with numerical results for both evaluated geometries as it is discussed below.

\subsection{1.-Thin rod}

The pure axial resonant modes of an elastic cylinder are multiples of the eigenfrequency associated with the first mode. Due to the symmetrical excitation the modes form a series of odd natural multiples of the fundamental frequency. Table 2 shows analytically derived values for the case of a cylinder with length $Z=6 \mathrm{~cm}$, and wave speed $3859 \mathrm{~m} / \mathrm{s}$ (coincident with the speed associated with the biggest $k^{D}$ coefficient, $c^{D}$ ) compared with numerical data. The piezoelectric is excited in open circuit condition so the eigenfrequencies are associated with antiresonances. 
Results are also shown in figure 3 where impedance is plotted against frequency in the region with highest signal to noise ratio. Excitation was achieved using Ricker wavelets with a Gaussian-like spectrum.

The total integration time at the experiment was $5 \mathrm{~ms}$, which implies a frequency resolution of $0.2 \mathrm{kHz}$. The spatial resolution is one-half the internodal distance and it generates an additional imprecision in the derived resonance frequencies of $0.8 \%$. The small differences found between numerical and analytical results can be attributed to this imprecision, and they are not suggesting a wrong behavior of the model.

\begin{tabular}{|l|l|l|l|l|l|}
\hline 16.5 & -4.78 & -8.45 & 0 & 0 & -274 \\
\hline-4.78 & 16.5 & -8.45 & 0 & 0 & -274 \\
\hline-8.45 & -8.45 & 20.7 & 0 & 0 & 593 \\
\hline 0 & 0 & 0 & 43.5 & 741 & 0 \\
\hline 0 & 0 & 0 & 741 & 2768 & 0 \\
\hline-274 & -274 & 593 & 0 & 0 & 3006 \\
\hline
\end{tabular}

Table 1: Piezoelectric properties of the material used in simulations, written in the matrix form: $\left(\begin{array}{cc}C^{E} & d \\ d & \varepsilon^{\sigma}\end{array}\right)$. Compliances units are $10^{-12} \mathrm{~m}^{2} \mathrm{~N}^{-1}$ and piezoelectrical charge constant units are $10^{-12} \mathrm{mV}^{-1}$. Relative permittivities.

\begin{tabular}{|l|l|l|l|l|}
\hline Numeric f(kHz) & 32.4 & 96.8 & 160.3 & 223.1 \\
\hline Analytic f(kHz) & 32.1 & 96.4 & 160.8 & 225.1 \\
\hline
\end{tabular}

Table 2: Numerical and analytical results for the first pure axial modes in a thin cylindrical rod with length, $\mathrm{Z}=60 \mathrm{~mm}$.

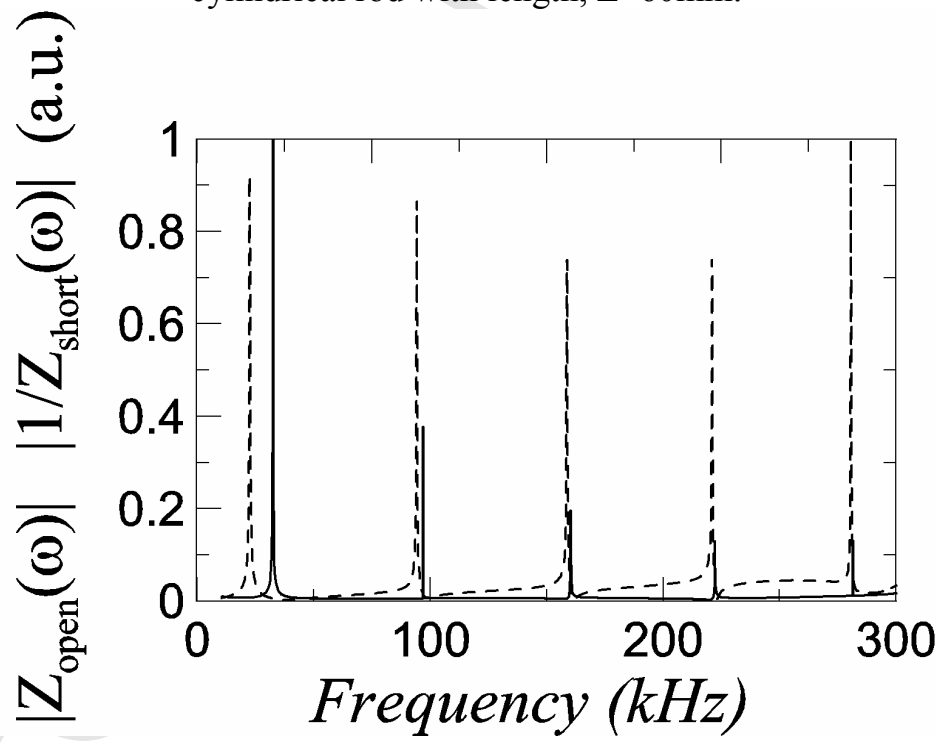

Figure 3.- Module of impedance in open circuit (continuous line), and module of admittance in short-circuit (dashed), for the case of a thin piezoelectric rod in arbitrary units. 


\subsection{2.-Thin disk}

Now let us consider the case of a thin disk. The resonance frequencies of the radial modes of a thick elastic cylinder in free-free-free conditions are given by the solutions of the implicit equation [18]

$$
J_{1}(\kappa R)=\beta \cdot \kappa R \cdot J_{2}(\kappa R)
$$

where $J_{1}$ and $J_{2}$ are Bessel functions of first and second order respectively, $k$ is the wave number, $R$ is the external radius of the cylinder, and beta is a dimensionless coefficient depending on elastic material properties.

In an isotropic case, beta is obtained as

$$
\beta=\frac{\lambda+2 G}{2(\lambda+G)}
$$

Here, $\lambda$ and $G$ are the Lamè coefficients and $\beta$ always takes a positive value smaller than one. In our case, this coefficient takes the value 0.7625 , so that the effective Young modulus associated with the calculation of wave speed is $656 \cdot 10^{10} \mathrm{Nm}^{-2}$, and the associated wave speed is:

$$
c_{L}=\sqrt{\frac{6 \cdot 56 \cdot 10^{10}}{7500}}=2959 \mathrm{~ms}^{-1} \text {. }
$$

With this data, one can change the solutions of Eq. (35) into resonant frequencies. This equation is solved when $k R=(2.056,5.392,8.532,11.733,14.884,18.033, \ldots)$ obtaining for $\mathrm{R}=4 \mathrm{~cm}$ the numerical eigenfrequencies shown in table 3 .

In this table a comparison is performed between analytical purely elastic results, and numerical results in conditions of shortcircuit. Results can also be observed in the figure 4, where admittance has been plotted against frequency in the region with highest signal to noise ratio. Again, excitation is carried out using Ricker wavelets with a Gaussianlike spectrum.

Very good agreement between analytical and numerical results is found for axial modes in open circuit condition (antiresonances) and for radial modes in short circuit (resonances). This particularity has been justified formerly in this section, but we are trying to extend the explanation of this point in the next lines:

The mechanical coefficients of compliance tensors, in piezoelectric materials, change depending on the electrical condition of constant $\vec{D}(t)$ or constant $\vec{E}(t)$. In a domain with strictly uniform and constant $\vec{D}(t)$ or $\vec{E}(t)$, the wave speed is a direct consequence of the value of coefficients $k^{D}$ and $k^{E}$ respectively. In the case of an axial mode in a thin rod, an open-circuit condition is accepted to approach a constant null displacement in any field line of the piezo, so that the wave speed is constant for any axial mode and can be found from the elastic coefficients (this wave speed is denoted $c^{D}$ ). However, if the same rod is excited in short-circuit, this implies null electrostatic potential which means that the average value of the electric field trough a field line is null into the substrate: the condition of null electric field is almost but not strictly fulfilled. Moreover it can be 
demonstrated that for low frequency modes with large value of $\lambda$, the wave speed will approach the theoretical speed associated with the null electric field condition $\left(c^{E}\right)$ but for high frequency modes (in shortcircuit) the wave speed will tend to be similar to $c^{D}$. This theoretical evidence is reflected in the numerical results of figure 3.

\begin{tabular}{|l|l|l|l|l|l|l|}
\hline Numeric f(kHz) & 24.2 & 63.6 & 100.9 & 137.9 & 174.2 & 210.2 \\
\hline Analytic f(kHz) & 24.2 & 63.5 & 100.4 & 138.1 & 175.2 & 212.3 \\
\hline
\end{tabular}

Table 3: Numerical and analytical results for the first pure radial modes in a thin disk with radius, $\mathrm{R}=40 \mathrm{~mm}$.

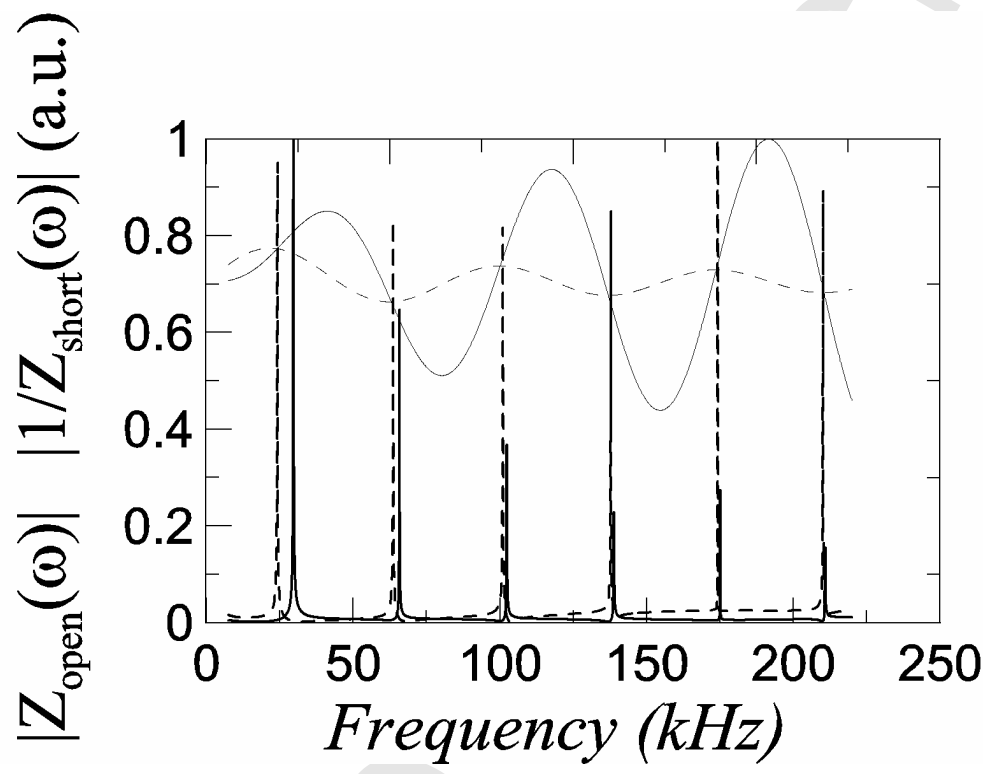

Figure 4.- Modules of impedance in open circuit (dashed line), and admittance in short-circuit (continuous), for the case of a thin piezoelectric disk in arbitrary units. The Bessel functions are plotted, by adding an arbitrary constant value to simplify visual inspection.

On the other hand, if we study now the case of a radial mode in a thin disk, the shortcircuit condition is accepted to approach a null electric field along any radial line within the substrate, and thus the wave speed will equal the theoretical speed $c^{E}$, which can be found from the elastic coefficients and is constant for any radial mode. However, if this disk is excited in open circuit, we have a condition of null total charge in the electrodes, and this means that the average value of the charge density into the electrodes - and the displacement field into the substrate - is null; the condition of null electric displacement is almost but not strictly fulfilled. It can be again demonstrated that, for low frequency modes the wave speed will approach the theoretical speed $c^{D}$, but for high frequency modes (in open circuit) the wave speed will tend to be similar to $c^{E}$. Numerical results agree with this assertion as shown in figure 4.

This particular property of transducer dynamics - that high frequency modes propagate with speed $c^{E}$ in radial directions and $c^{D}$ in axial directions, independent of electrical contour conditions - is also found in general three dimensional substrates. Obtaining the 
analytical value of all eigenfrequencies in three dimensional substrates is not an easy task. In the limit cases of low- and high-frequency modes, we can forecast the wave speed; However, in the "middle frequencies region" we only can affirm initially that, independent of electrical contour conditions, the eigenfrequencies will be found somewhere between those predicted for elastic materials with similar shape and with wave speeds similar to $c^{D}$ and $c^{E}$.

To determine analytically the eigenfrequencies associated with a particular mode, we have to know the wave speed associated with that particular vibratory state. An analysis of this situation seems to drive to a significant conclusion: in the mid-frequency range (where wavelengths are small compared to physical dimensions) the wave speed may vary as a function of position, within the range defined by the limit values $c^{D}$ and $c^{E}$. This spatial dependence of wave speed has an interesting consequence related to the Fermat principle: the trajectory of an energy ray could be curved. This assertion is compatible with the presence of solitons in linear piezoelectrics, and is also compatible with the results found in our simulations, as shown in figure 5. In fact, if the diameter of the cylindrical rod is modified, the eigenfrequency associated with the main axial mode remains constant, but for the second pure axial mode, the eigenfrequency decreases as radial dimension increases. This decrease of the eigenfrequency is associated with a reduction of the wave speed in this mode, as predicted by the theory. The bigger the radius, the bigger the variation of electrical displacement in the piezoelectric substrate (even in open circuit conditions), and the smaller the average Young modulus and wave speed.

The numerical robustness of the model was finally tested by changing spatial mesh resolution, total integration time, Courant number in the region defined by the stability criterion, and excitation function. For the sake of brevity, all these tests are not presented here. However, we can stand that no significant differences were found.

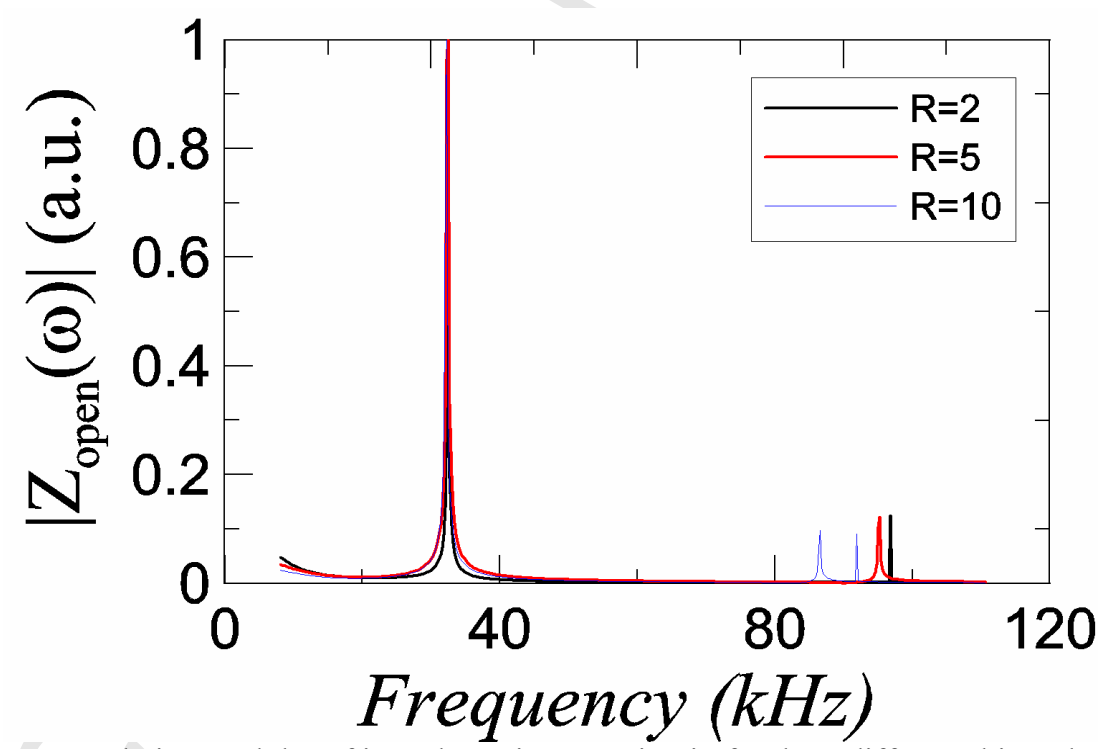

Figure 5.- Relative modules of impedance in open circuit, for three different thin rods, with length $\mathrm{Z}=6 \mathrm{~cm}$, and respective radius $\mathrm{R}=2 \mathrm{~mm}, \mathrm{R}=5 \mathrm{~mm}$ and $\mathrm{R}=10 \mathrm{~mm}$ 


\section{2.- Spatial and time response}

Previous section illustrates good agreement with theory in the evaluation of the system response in frequency domain. As the calculations are performed in the time domain, a good accuracy in frequency implies necessarily a good accuracy in the time response. In this section, and with the aim of illustrating wave propagation, we show a set of spatial instantaneous plots.

In figure 6 three different instants of propagation of a high frequency Ricker pulse are plotted. Figure 7 illustrates important differences associated with the acceptance of transversal non-uniformity for the displacement field. The two plots of fig.7 are synchronous and the excitation function is similar, but in the first one we have an open circuit condition, and in the second we have a constant electric displacement condition. In several previous models these conditions are confused, using $D=0$ as a simplification of the real condition $I=0$. Experiments suggest that this simplification leads to considerably different results. Here we show instantaneous spatial images, but frequency responses are also different.

Finally, figure 8 plots the cumulative quadratic axial displacement of a thin rod in its second pure axial mode. One may appreciate that the amplitude of the perturbation is not constant in a radial direction as it happens in extremely thin rods. This energy concentration along the axis is associated with a reduction of the effective modal wave speed, as commented in the frequency response section.

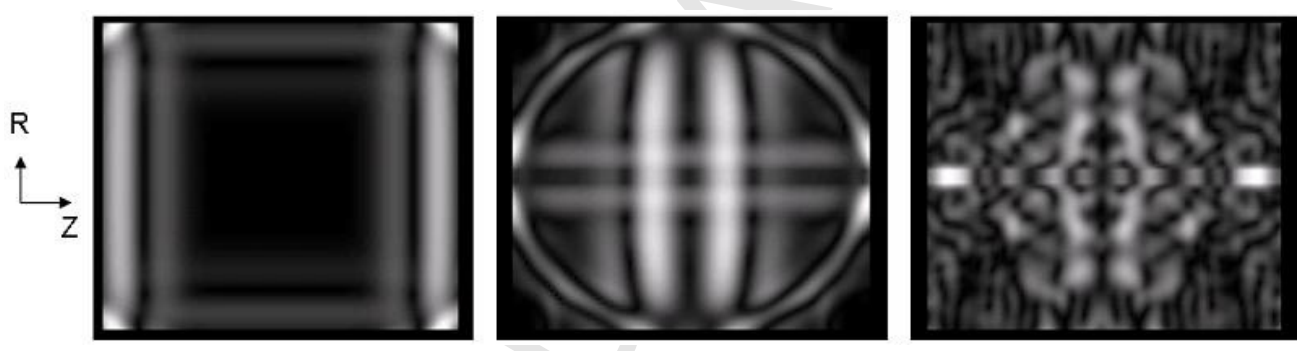

Figure 6.- Volumetric compression in RZ plane. Snapshots at three different instants $\left(t_{1}=100 \mathrm{~ns}\right.$, $\left.t_{2}=300 \mathrm{~ns}, t_{3}=50 \mu \mathrm{s}\right)$ of a $2 \mathrm{MHz}$ frequency pulse propagating into a cylindrical piezoelectric electrically excited in its flat electrodes with free mechanic boundary conditions $(\mathrm{R}=6.5 \mathrm{~mm}$, $\mathrm{Z}=15 \mathrm{~mm}$, Ricker-wavelet pulse function for electrostatic potential).

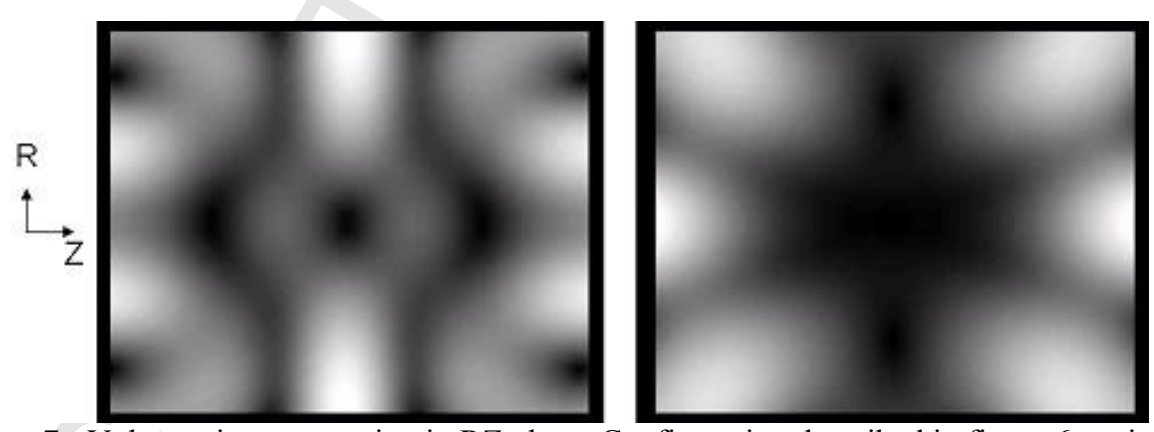

Figure 7.- Volumetric compression in RZ plane. Configuration described in figure 6 excited, in open circuit, in its second anti-resonant mode (left) and synchronous image, modeled similarly, in conditions of strict uniform electrical displacement (right) 


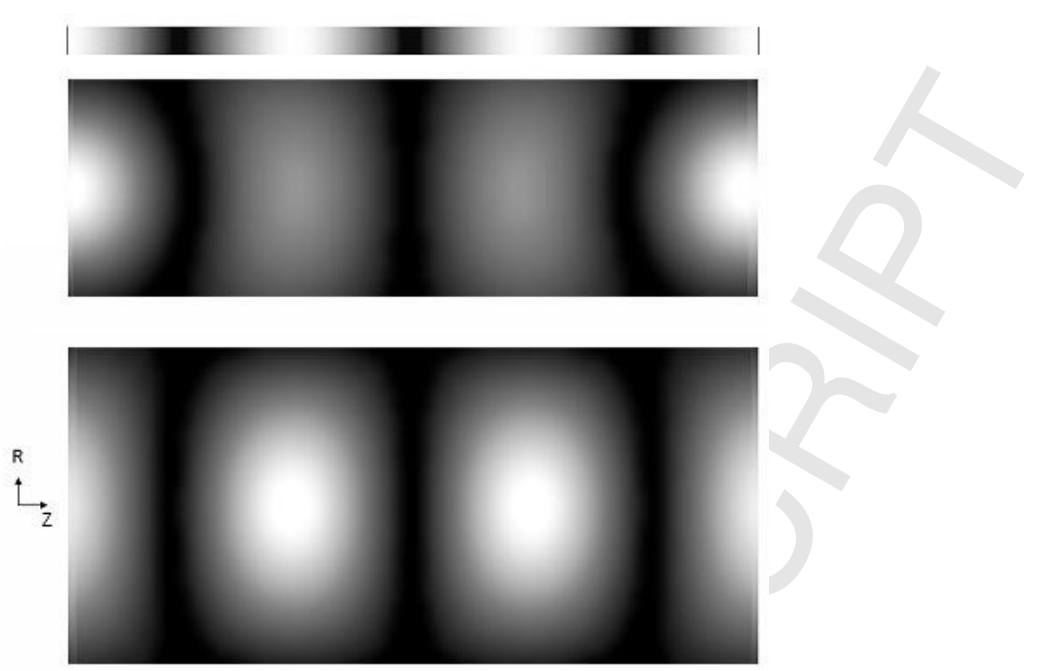

Figure 8.- Instantaneous quadratic axial speed in cylinders $(Z=60 \mathrm{~mm}$ and $\mathrm{R}=2,10,14 \mathrm{~mm})$, excited in their second antiresonant mode.

\section{7- CONCLUSIONS}

As established in the introduction, the aim of this work is to develop a model which is on the one hand fully explicit, and on the other hand which agrees with real physics in formulation and results.

The explicitness of the model has been fully satisfied, even when this requires a small change in the system of equations depending on the kind of electrical excitation applied. One of the equations requires a spatial integration; but, even in this case, no matrix inversions are performed, and all model equations are explicit.

The discretisation of the system is performed by means of a grid that is fully staggered in space and in time, where no high order integrations are required. Then, all the discrete system equations are centered, and no other approaches are used in the integration (such as Runge-Kutta). Accuracy is then determined by numerical precision, and by the validity of the substitution of mathematical differentials by finite differences. Therefore, respecting soft criteria of mesh size as a function of the minimum wavelength to be simulated, and the necessary stability criterion, the system behaves satisfactorily. Moreover, the form adopted for the canonic piezoelectrical equations improves stability: since the stability criterion depends directly on the wave speed, then we have chosen the set of equations as a function of $k^{D}$ coefficients directly related to the $c^{D}$ speed, which is the faster of both characteristic piezoelectric speeds. This means that the "natural" speed of our system equations is $c^{D}$, whereas $c^{E}$ is the consequence of the interference between fully mechanical and piezoelectric coupled dynamics. It is easy to demonstrate that enforcing the electric displacement to be null in each integration step, the system reduces exactly to a simple elastodynamic case with wave speed $c^{D}$ coincident with that of Schubert [16]. Furthermore, the electrical part of the system can be formally accepted as a set of volumetric source terms added to the purely mechanical part. These source terms can only decrease the wave speed, which warranties stability. 
Comparison of numerical results with known analytical solutions shows good agreement. In fact, only two simplifications of 'real' physics have been considered. The first is the quasistatic approach which is more acceptable the bigger is the ratio between electromagnetic and mechanical wave speeds. Since these speeds are respectively in the order of $10^{8} \mathrm{~m} / \mathrm{s}$ and $10^{3} \mathrm{~m} / \mathrm{s}$, this approach is reasonably acceptable. The second assumption introduced in this work, is that the electric displacement is reduced to parallel field lines in the piezoelectric substrate, with no flow going out of it.

The presented model is particularly adequate to simulate transient response of transducers, during the external excitation by narrow band pulses close to the axial lowest modes (as justified in appendix B), but in the results section it has been shown that for radial modes, and evaluating the system stationary response, the results are also acceptable.

Therefore, we can conclude that our aim has been satisfied. However, further improvements both in electromagnetic simplifications and in mechanical properties of the material, like dissipation, nonlinear plastic hysteresis, and so on, remain interesting areas for development.

\section{8.- ACKNOWLEDGEMENTS}

The authors want to thank Miquel Ardid, Víctor Sánchez and Bernardino Roig for their cooperation and fruitful discussions. This study was supported by the Programa de Apoyo a la Investigación y Desarrollo PAID-06-10-002-295 of Universidad Politécnica de Valencia.

\section{9.- BIBLIOGRAPHY}

[1] S. Kostek, C. J. Randall. Modeling of a piezoelectric transducer and its application to full waveform acoustic logging. J. Acoust. Soc. Am. 1994; 95: 109-122

[2] Smith PM, Ren W. Finite-difference time-domain techniques for SAW device Analysis. IEEE Ultrasonics Symposium 2002; 326.

[3] Chagla F, Smith PM. Finite-difference time-domain techniques methods for piezoelectric Crystals. IEEE Transactions on Ultrasonics, Ferroelectrics, and Frequency Control 2006; 53: 10.

[4] Wong KY, Tam WY. Finite-difference time-domain simulation of dispersive layered SAW filters including electrode massloading. IEEE Ultrasonics symposium 2005; 1568-1571

[5] Gsell D, Dual J. Simulation of elastic wave propagation in cylindrical structures including excitation by piezoelectric transducers. Ultrasonics 2002; 40: 181-186

[6] May F.; Dual, J. Focusing of pulses in axially symmetric elastic tubes with fluid filling and piezo actuator by a finite difference simulation and a method of time reversal. Wave Motion 2006, 43: 311-322

[7] Yamada Y, Sato M. Application of extended finite-difference time-domain method to twodimensional dynamic analysis of a piezoelectric vibrator. Japanese Journal of Applied Physics 1998; 37: 255-256. 
[8] Sato M. Numerical formulation of the FDTD method for transient analysis of elastic wave fields in the Y-Z plane of quartz. Japanese Journal of Applied Physics 2005; 44: 4490-4493

[9] Hornsby JS, and Das-Gupta DK. Finite-difference modeling of piezoelectric composite transducers. Journal of Applied Physics 2000; 87: 467-473

[10] Chagla F, Cabani C, Smith PM. Perfectly matched layer for FDTD computations in piezoelectric crystals. Proc. IEEE Ultrason. Symp., 2004: 517-520.

[11] Sladek J, Sladek V, Solek P, Saez A. Dynamic 3D axisymmetric problems in continuously non-homogeneous piezoelectric solids. International Journal of Solids and Structures 2008; 45: 4523-4542

[12] Samal MK, Seshu P, Parashar SK, Von Wagner U, Hagedorn P, Dutta BK, Kushwaha HS. Nonlinear behaviour of piezoceramics under weak electric fields Part-I: 3-D finite element formulation. International Journal of Solids and Structures 2006; 43: 1422-1436

[13] Wu DH, Chien WT, Yang CJ, Yen YT. Coupled-field analysis of piezoelectric beam actuator using FEM. Sensors and Actuators A 2005; 118: 171-176

[14] Brodal E, Hesthaven JS, Melandso F. Numerical modeling of double-layered piezoelectric transducer systems using a high-order discontinuous Galerkin method. Computers and Structures 2008; 86: 1747-1756

[15] Qin TY, Yu YS, Noda NA, Finite-part integral and boundary element method to solve three-dimensional crack problems in piezoelectric materials. International Journal of Solids and Structures 2007; 44: 4770-4783

[16] Schubert F, Peiffer A, Köhler B. The elastodynamic finite integration technique for waves in cylindrical geometries. Journal of the Acoustical Society of America 1998; 104: 2604-2614.

[17] Taflove A, Hagness SC. Computational Electrodynamics: The Finite-Difference TimeDomain Method. 3rd ed. Norwood, MA: Artech House, 2005.

[18]Nieves FJ, Gascón F, Bayón A. An analytical, numerical, and experimental study of the axisymmetric vibrations of a short cylinder. Journal of Sound and Vibration 2008; 313: 617630 


\section{APPENDIX A}

\section{A.1.- Explicit discrete equations}

The explicit equations for the algorithm are presented here for a quadratic grid. The variable names are rewritten avoiding sub index, so the instantaneous punctual value of a variable, for example $\sigma_{1}\left(r_{j}, z_{k}, t_{n}\right)$ is denoted as $\sigma 1_{j, k}^{n}$ where $r_{j}=(j-1) \Delta r$, $z_{k}=(k-1) \Delta z$, and $t_{n}=n \Delta t$. It must be also remarked that the physical and computational domain differ one from each other (by half spatial step in each boundary). The physical edge is defined by the node-line that satisfies strictly the boundary conditions; for example, in the case considered of stress-free conditions, the axial stress $\sigma 3$ will be null for $\mathrm{z}=0$ and $\mathrm{z}=\mathrm{Z}$, and the physical domain shall start and finish in nodes of the variable $\sigma 3$, respectively, as shown in figure 1 . The relations between the physical and computational limits are given by

$$
Z=\Delta r(K-2), \quad R=\Delta r(J-1)
$$

The FDTD scheme is based on a centered staggered grid, but in some equations the value of a particular stress or velocity component is required out of its self node, then linear interpolation is used, for example

$$
\sigma 3_{j, k+1 / 2}^{n}=\frac{\sigma 3_{j+1 / 2, k+1 / 2}^{n}+\sigma 3_{j-1 / 2, k+1 / 2}^{n}}{2}
$$

Definitely, assuming previous general considerations, dynamic equations are

$$
u r_{j, k+1 / 2}^{n+1 / 2}=\left\{\begin{array}{l}
u r_{j, k+1 / 2}^{n-1 / 2}+\frac{\Delta t}{\rho_{o} r_{j, k+1 / 2} \Delta r}\left(r_{j+1 / 2, k+1 / 2} \sigma 1_{j+1 / 2, k+1 / 2}^{n}-r_{j-1 / 2, k+1 / 2} \sigma 1_{j-1 / 2, k+1 / 2}^{n}\right) \\
+\frac{\Delta t}{\rho_{o} \Delta z}\left(\sigma 5_{j, k+1}^{n}-\sigma 5_{j, k}^{n}\right)+\frac{\Delta t}{2 \rho_{o} r_{j, k+1 / 2}}\left(\sigma 2_{j+1 / 2, k+1 / 2}^{n}+\sigma 2_{j-1 / 2, k+1 / 2}^{n}\right)
\end{array}\right\}
$$

for $j=1, \ldots, J-1$, and $k=1, \ldots, K-1$,

$$
u z_{j+1 / 2, k}^{n+1 / 2}=\left\{\begin{array}{l}
u z_{j+1 / 2, k}^{n-1 / 2}+\frac{\Delta t}{\rho_{o} r_{j+1 / 2, k} \Delta r}\left(r_{j+1, k} \sigma 5_{j+1, k}^{n}-r_{j, k} \sigma 5_{j, k}^{n}\right) \\
+\frac{\Delta t}{\rho_{o} \Delta z}\left(\sigma 3_{j+1 / 2, k+1 / 2}^{n}-\sigma 3_{j+1 / 2, k-1 / 2}^{n}\right)
\end{array}\right\}
$$

for $j=1, \ldots, J-1$, and $k=2, \ldots, K-1$,

The constitutive axial equations for the next half-time step, are: 


$$
\begin{gathered}
\sigma 1_{j+1 / 2, k+1 / 2}^{n+1}=\left\{\begin{array}{l}
\sigma 1_{j+1 / 2, k+1 / 2}^{n}+\frac{k 11 \Delta t}{\Delta r}\left(u r_{j+1, k+1 / 2}^{n+1 / 2}-u r_{j, k+1 / 2}^{n+1 / 2}\right)+k 12 \Delta t \frac{u r_{j+1, k+1 / 2}^{n+1 / 2}+u r_{j, k+1 / 2}^{n+1 / 2}}{2 r_{j+1 / 2, k+1 / 2}} \\
+\frac{k 13 \Delta t}{\Delta z}\left(u z_{j+1 / 2, k+1}^{n+1 / 2}-u z_{j+1 / 2, k}^{n+1 / 2}\right)-h 13\left(D 3_{j+1 / 2, k+1 / 2}^{n+1}-D 3_{j+1 / 2, k+1 / 2}^{n}\right)
\end{array}\right\} \\
\sigma 2_{j+1 / 2, k+1 / 2}^{n+1}=\left\{\begin{array}{l}
\sigma 2_{j+1 / 2, k+1 / 2}^{n}+\frac{k 12 \Delta t}{\Delta r}\left(u r_{j+1, k+1 / 2}^{n+1 / 2}-u r_{j, k+1 / 2}^{n+1 / 2}\right)+k 11 \Delta t \frac{u r_{j+1, k+1 / 2}^{n+1 / 2}+u r_{j, k+1 / 2}^{n+1 / 2}}{2 r_{j+1 / 2, k+1 / 2}} \\
+\frac{k 13 \Delta t}{\Delta z}\left(u z_{j+1 / 2, k+1}^{n+1 / 2}-u z_{j+1 / 2, k}^{n+1 / 2}\right)-h 13\left(D 3_{j+1 / 2, k+1 / 2}^{n+1}-D 3_{j+1 / 2, k+1 / 2}^{n}\right)
\end{array}\right\} \\
\sigma 3_{j+1 / 2, k+1 / 2}^{n+1}=\left\{\begin{array}{l}
\sigma 3_{j+1 / 2, k+1 / 2}^{n}+\frac{k 13 \Delta t}{\Delta r}\left(u r_{j+1, k+1 / 2}^{n+1 / 2}-u r_{j, k+1 / 2}^{n+1 / 2}\right)+k 13 \Delta t \frac{u r_{j+1, k+1 / 2}^{n+1 / 2}+u r_{j, k+1 / 2}^{n+1 / 2}}{2 r_{j+1 / 2, k+1 / 2}} \\
+\frac{k 33 \Delta t}{\Delta z}\left(u z_{j+1 / 2, k+1}^{n+1 / 2}-u z_{j+1 / 2, k}^{n+1 / 2}\right)-h 33\left(D 3_{j+1 / 2, k+1 / 2}^{n+1}-D 3_{j+1 / 2, k+1 / 2}^{n}\right)
\end{array}\right\}
\end{gathered}
$$

for $j=1, \ldots, J-1$, and $k=1, \ldots, K-1$,

For tangential equations, we have

$$
\sigma 5_{j, k}^{n+1}=\sigma 5_{j, k}^{n}+\frac{k 55 \Delta t}{2 \Delta z}\left(u r_{j, k+1 / 2}^{n+1 / 2}-u r_{j, k-1 / 2}^{n+1 / 2}\right)+\frac{k 55 \Delta t}{2 \Delta r}\left(u z_{j+1 / 2, k}^{n+1 / 2}-u z_{j-1 / 2, k}^{n+1 / 2}\right)
$$

for $j=1, \ldots, J-1$, and $k=2, \ldots, K-1$,

And finally, the first step of the algorithm, where a known electric potential source term $\phi(t)$ is taken into account, is

$$
D 3_{j+1 / 2}^{n+1}=D 3_{j+1 / 2}^{n}+\frac{\Delta t}{\beta 33} \sum_{k=1}^{K-1} p m e c_{j+1 / 2, k+1 / 2}^{n+1 / 2}-\frac{1}{Z \beta 33}\left(\phi_{j+1 / 2}^{n+1}-\phi_{j+1 / 2}^{n}\right)
$$

where $\mathrm{Z}$ is the size of the physical domain, in this case $Z=\Delta r(K-2)$, and the punctual contribution of the mechanic deformation to the time derivative of the electric field, pmec $_{j+1 / 2, k+1 / 2}^{n+1 / 2}$, is

$$
\operatorname{pec}_{j+1 / 2, k+1 / 2}^{n+1 / 2}=\left\{\begin{array}{l}
\frac{h 13}{\Delta r}\left(u r_{j+1, k+1 / 2}^{n+1 / 2}-u r_{j, k+1 / 2}^{n+1 / 2}\right)+h 13 \frac{u r_{j+1, k+1 / 2}^{n+1 / 2}+u r_{j, k+1 / 2}^{n+1 / 2}}{2 r_{j+1 / 2, k+1 / 2}} \\
+\frac{h 33}{\Delta z}\left(u z_{j+1 / 2, k+1}^{n+1 / 2}-u z_{j+1 / 2, k}^{n+1 / 2}\right)
\end{array}\right\}
$$

for $j=1, \ldots, J-1$, 


\section{A.2.- Boundary conditions}

\section{A.2.1.- Axial boundary conditions}

Because of cylindrical symmetry both compounds $u_{r}$ and $\sigma_{r z}$ vanish at the axis; therefore, for stability reasons, it is more convenient to place at the axis the nodes associated to these variables. As a consequence no field component has to be calculated at the axis, and the numerical problems involving the wave propagation in these singular points are avoided. The equation of this condition reads:

$$
\begin{array}{ll}
\sigma 5_{1, k}=0, & \text { for } k=1, \ldots, K, \\
u r_{1, k+1 / 2}=0, & \text { for } k=1, \ldots, K-1,
\end{array}
$$

\section{A.2.2.- Stress-free boundary conditions}

As the model describes piezoelectric actuators, only the stress-free conditions are evaluated in Section 6 -Numerical Results and Discussion. However, the piezoelectric domain can be evaluated by considering fixed boundaries or by charging to a different medium (the completely clamped situation has no sense here because the system stays in the trivial solution of no movement when excited electrically).

Boundary conditions are defined for the first node lines and for the virtual non-physic lines as follows: i) Tangential stressed are null, ii) the axial stress second components $\left(\sigma_{\varphi}\right)$ is linearly extrapolated in the virtual node lines from the value of two attached nodes, and iii) other axial stresses in the virtual node lines are imposed to have the opposite value of the attached node line, so the average evaluated in the real physical perimeter is null. Finally, iv) no conditions are required for speed and electrical vectors in the extreme physical node lines, as they are computed by means of main equations considering the values of the virtual nodes. The particular expressions of these conditions are noted as follows:

Free condition in the electrodes $(z=0, z=Z)$

Non-physical nodes:

$$
\begin{aligned}
& \sigma 3^{n}{ }_{j+1 / 2,1 / 2}=-\sigma 3^{n}{ }_{j+1 / 2,1+1 / 2} \\
& \sigma 3^{n}{ }_{j+1 / 2, K+1 / 2}=-\sigma 3_{j+1 / 2, K-1 / 2}^{n} \quad \text { for } j=1, \ldots, J-1,
\end{aligned}
$$

Conditions at the physical edge for tangential stress

$$
\begin{array}{ll}
\sigma 5_{j, 1}^{n}=0 & \\
\sigma 5_{j, K}^{n}=0 & \text { for } j=1, \ldots, J
\end{array}
$$

No conditions are required for the rest stress components

Free condition in $r=R$

Physical edge:

$$
\sigma 5_{J, k}^{n}=0 \quad \text { for } k=1, \ldots, K
$$

Non physical nodes:

$$
\begin{aligned}
& \sigma 1_{J+1 / 2, k+1 / 2}^{n}=-\sigma 1_{J-1 / 2, k+1 / 2}^{n} \\
& \sigma 2_{J+1 / 2, k+1 / 2}^{n}=2 \cdot \sigma 2_{J-1 / 2, k+1 / 2}^{n}-\sigma 2_{J-3 / 2, k+1 / 2}^{n} \quad \text { for } k=1, \ldots, K-1,
\end{aligned}
$$

No conditions are required for the rest stress components 


\section{APPENDIX B}

In the present appendix several arguments are presented in order to justify the approach introduced by equations (8) and (9). The assumption is based on the condition of constant displacement field through an axial line, and permits to obtain the physical variables in an explicit scheme.

The approach, as will be shown, is accurate to model the transient response of transducers, during the external excitation by narrow band pulses close to the resonant lower modes. In order to prove the validity of the approach an ideal situation is initially shown. More complex cases are presented to approach to real cases. Finally a numerical evaluation is presented to illustrate the validity of the approach.

\section{B.1.- Ideal constant excitation}

During the excitation, the flat circular charged electrodes generate an electric field. By considering a constant excitation, the complete ceramic is uniformly polarized (as shown in figure B.1. The electric and displacement field have the same distribution, being parallel one to each other at any point. As it can be appreciated, the radial component of both electric fields, $\boldsymbol{E}$ and $\boldsymbol{D}$, is notably smaller than the axial one, and thus they can be neglected in the first approach in the center of the capacitor. The approach $D(r)=0$ is better, the better is the relation $\mathrm{R} / \mathrm{Z}$

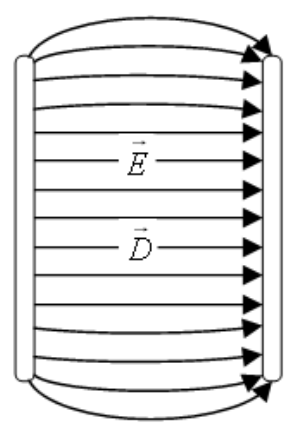

Figure B.1.- Field lines $(\boldsymbol{E}$ and $\boldsymbol{D})$ in a charged flat capacitor in vacuum

\section{B.2.- Instantaneous excitation in axial mode}

Consider a piezoelectric ceramic excited in an axial mode. We focus on an instant in which a non-null external charge is applied at the electrodes. In this case, the wavefronts are accepted to be parallel to the electrodes. Each wavefront has a different compressive state, so each one can be considered a different dielectric layer, being the boundaries of this layers perpendicular to the electric and displacement field. Due to this perpendicularity, electric field changes in value but not in direction between contiguous layers. Displacement field - according to Gauss' law $\nabla \cdot \vec{D}=\rho$ - remains constant through a field line, as stated in our hypothesis.

\section{B.3.- Real instantaneous situation}


Out of an axial mode, each point of the substrate has a different instantaneous state, so perpendicular boundaries cannot be defined, and the electric and displacement field change their direction and value through all the substrate. However there are real cases similar to the ideal hypothesis, such as the following (Justification of the truthfulness of these statements placed in next section):

a) During the excitation: If an external instantaneous electric fields is being applied, the field lines will follow the distribution plotted in figure B.1 more accurately than if the electric field is exclusively generated by the punctual piezoelectric stresses.

b) If the excitation frequency is close to any axial mode: Then the energy is accumulated in these modes and mechanical wavefronts are almost perpendicular to the electric field. The dielectric properties will also change in radial direction bending the field lines, but the relevant variations are induced in the axial direction

c) For lower modes, the instantaneous dielectric properties change only a few times over the space among their extreme values. If dielectric properties change smoothly, the field lines get smoothly curved

\section{B.4.- Simulation in real situation}

To test the validity of our hypothesis, several simulations have been performed by calculating both electric field components from constitutive equations, with the purpose of obtaining the ratio $\tan (\theta)=E_{r} / E_{z}$ for each computational node. Transducers with different shapes are excited by different frequencies and the hypothesis is proven to be reasonably adequate. Next figure B.2 illustrates an example. Figure B.2a represents, for each computational step, the median in the whole computational domain of the punctual value $\tan (\theta)=E_{r} / E_{z}$. The continuous line represents instantaneous values, and the discontinuous line draws the average of this median during the previous computed time. It can be noted that the function converges to a value smaller than 0.1 , what implies that the angle is smaller than $6^{\circ}$.

It can be appreciated that, periodically the value of the median angle increases. This growth coincides with the instants for which the voltage between the electrodes is null in the whole domain (see figure B.2b). In these instants the average value of $E_{z}$ in a field line is null and big ratios of $\tan (\theta)=E_{r} / E_{z}$ are associated to numerical imprecision at the points with smaller values of $E_{z}$.

During the excitation, the typical values of $\tan (\theta)=E_{r} / E_{z}$ are small, and they increase with time after the excitation, when the energy still keeps into the transducer due to the non-dissipative boundary conditions defined. In a real situation, the transducer radiates and dissipates energy, therefore the most relevant results of the simulation are those induced during the excitation.

Simulations performed in different conditions show that the convergence values of the "median angle" (discontinuous line in figure B.2a) depend on the frequency of the excitation, the shape of the piezoelectric substrate, and the spectral distribution of the excitation signal. Generally, the smaller the frequency and the narrower the signal broadband, the better is the approximation of the hypothesis. Several cases in which the hypothesis is less accurate have also been tested. The convergence value of the median 
of $\tan (\theta)$ always keeps under the value 0.35 , which implies that the angles are smaller than $20^{\circ}$ for the worst cases.
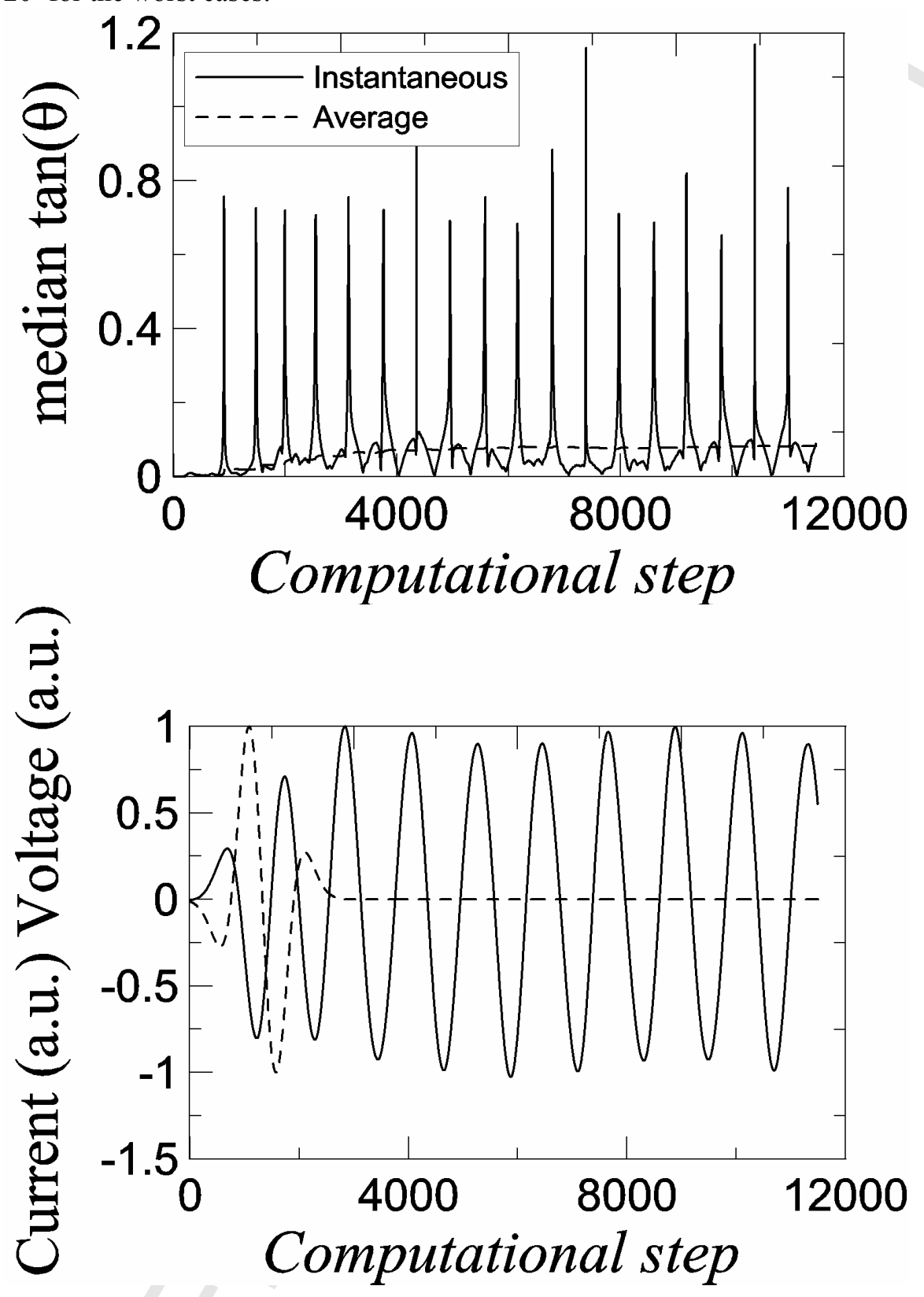

Figure B.2a.-Instantaneous median of the value $\tan (\theta)=E_{r} / E_{z}$ for the whole computational domain (continuous); and temporary average of this median (dashed). Figure B.2b.- Electric current (dashed) and voltage between electrodes (continuous)

To sum up, the model is reasonably accurate to simulate transient responses excited by pulses with central frequency close to the resonance eigenfrequencies of the first resonant modes. 


\section{TABLES AND TABLE CAPTIONS}

Table 1: Piezoelectric properties of the material used in simulations, written in the matrix form: $\left(\begin{array}{cc}C^{E} & d \\ d & \varepsilon^{\sigma}\end{array}\right)$. Compliances units are $10^{-12} \mathrm{~m}^{2} \mathrm{~N}^{-1}$ and piezoelectrical charge constant units are $10^{-12} \mathrm{mV}^{-1}$. Relative permittivities,

Table 2: Numerical and analytical results for the first pure axial modes in a thin cylindrical rod with length, $Z=60 \mathrm{~mm}$.

Table 3: Numerical and analytical results for the first pure radial modes in a thin disk with radius, $\mathrm{R}=40 \mathrm{~mm}$. 


\begin{tabular}{|l|l|l|l|l|l|}
\hline 16.5 & -4.78 & -8.45 & 0 & 0 & -274 \\
\hline-4.78 & 16.5 & -8.45 & 0 & 0 & -274 \\
\hline-8.45 & -8.45 & 20.7 & 0 & 0 & 593 \\
\hline 0 & 0 & 0 & 43.5 & 741 & 0 \\
\hline 0 & 0 & 0 & 741 & 2768 & 0 \\
\hline-274 & -274 & 593 & 0 & 0 & 3006 \\
\hline
\end{tabular}

Table 1: Piezoelectric properties of the material used in simulations. written in the matrix form: $\left(\begin{array}{cc}C^{E} & d \\ d & \varepsilon^{\sigma}\end{array}\right)$. Compliances units are $10^{-12} \mathrm{~m}^{2} \mathrm{~N}^{-1}$ and piezoelectrical charge constant units are $10^{-12} \mathrm{mV}^{-1}$. Relative permittivities. 


\begin{tabular}{|l|l|l|l|l|}
\hline Numeric f(kHz) & 32.4 & 96.8 & 160.3 & 223.1 \\
\hline Analytic f(kHz) & 32.1 & 96.4 & 160.8 & 225.1 \\
\hline
\end{tabular}

Table 2: Numerical and analytical results for the first pure axial modes in a thin cylindrical rod with length, $Z=60 \mathrm{~mm}$. 


\begin{tabular}{|l|l|l|l|l|l|l|}
\hline Numeric $\mathrm{f}(\mathrm{kHz})$ & 24.2 & 63.6 & 100.9 & 137.9 & 174.2 & 210.2 \\
\hline Analytic f(kHz) & 24.2 & 63.5 & 100.4 & 138.1 & 175.2 & 212.3 \\
\hline
\end{tabular}

Table 3: Numerical and analytical results for the first pure radial modes in a thin disk with radius, $\mathrm{R}=40 \mathrm{~mm}$. 


\section{FIGURE CAPTIONS}

Figure 1.- Structure of the staggered spatial mesh

Figure 2.- Time and computational evolution of the simulation.

Figure 3.- Module of impedance in open circuit (continuous line), and module of admittance in short-circuit (dashed), for the case of a thin piezoelectric rod in arbitrary units.

Figure 4.- Modules of impedance in open circuit (dashed line), and admittance in short-circuit (continuous), for the case of a thin piezoelectric disk in arbitrary units. The Bessel functions are plotted, by adding an arbitrary constant value to simplify visual inspection.

Figure 5.- Relative modules of impedance in open circuit, for three different thin rods, with length $\mathrm{Z}=6 \mathrm{~cm}$, and respective radius $\mathrm{R}=2 \mathrm{~mm}, \mathrm{R}=5 \mathrm{~mm}$ and $\mathrm{R}=10 \mathrm{~mm}$

Figure 6.- Volumetric compression in RZ plane. Snapshots at three different instants $\left(t_{1}=100 n s\right.$, $\left.t_{2}=300 \mathrm{~ns}, t_{3}=50 \mu \mathrm{s}\right)$ of a $2 \mathrm{MHz}$ frequency pulse propagating into a cylindrical piezoelectric electrically excited in its flat electrodes with free mechanic boundary conditions $(\mathrm{R}=6.5 \mathrm{~mm}$, $\mathrm{Z}=15 \mathrm{~mm}$, Ricker-wavelet pulse function for electrostatic potential).

Figure 7.- Volumetric compression in RZ plane. Configuration described in figure 6 excited, in open circuit, in its second anti-resonant mode (left) and synchronous image, modeled similarly, in conditions of strict uniform electrical displacement (right)

Figure 8.- Instantaneous quadratic axial speed in cylinders $(Z=60 \mathrm{~mm}$ and $R=2,10,14 \mathrm{~mm})$, excited in their second antiresonant mode.

Figure B.1.- Field lines $(\boldsymbol{E}$ and $\boldsymbol{D})$ in a charged flat capacitor in vacuum

Figure B.2a.-Instantaneous median of the value $\tan (\theta)=E_{r} / E_{z}$ for the whole computational domain (continuous); and temporary average of this median (dashed).

Figure B.2b.- Electric current (dashed) and voltage between electrodes (continuous) 